\title{
The proof of three power-exponential inequalities
}

\section{Aníbal Coronel ${ }^{*}$ and Fernando Huancas}

${ }^{*}$ Correspondence:

acoronel@ubiobio.cl

GMA, Departamento de Ciencias

Básicas, Facultad de Ciencias,

Universidad del Bío-Bío, Campus

Fernando May, Chillán, Chile

\begin{abstract}
In this paper we prove three power-exponential inequalities for positive real numbers. In particular, we conclude that this proofs give affirmatively answers to three, until now, open problems (Conjectures 4.4, 2.1 and 2.2) posed by Cîrtoaje (J. Inequal. Pure Appl. Math. 10:21, 2009; J. Nonlinear Sci. Appl. 4(2):130-137, 2011). Moreover, we present a new proof of the inequality $a^{r a}+b^{r b} \geq a^{r b}+b^{r a}$ for all positive real numbers $a$ and $b$ and $r \in[0, e]$. In addition, three new conjectures are presented.
\end{abstract}

Keywords: power inequalities; exponential inequalities; power-exponential inequalities

\section{Introduction}

The power-exponential functions have useful applications in mathematical analysis and in other theories like statistics [1], biology [2,3], optimization [4], ordinary differential equations [5], and probability [6]. In recent years there has been intensive research in this area; see for instance [7-17] and the recent overview on general mathematical inequalities done by Cerone and Dragomir [18]. Some problems look like very simple but are difficult. For instance, we have the following two classical problems: find the solution of the equation $z e^{z}=a$ and the basic problem of comparing $a^{b}$ and $b^{a}$ for all positive real numbers $a$ and $b$. The first problem is perhaps one of the most ancient and useful problems concerning to power-exponential functions; see for instance [19-21]. It was introduced by Lambert in [22] and has been studied by recognized mathematicians like Euler, Pólya, Szegö, and Knuth; see [23-25]. The solution to the problem has inspired the definition of the well-known $W$-Lambert function; see [26]. For the solution to the second problem, see the discussion given in $[27,28]$ and more recently in [16]. Moreover, in spite of its algebraic simplicity, both problems are the central topic of a large number of research papers in the last years (see $[7,11,13]$ and references therein). In particular, in this paper, we are interested in some inequalities conjectured by Cîrtoaje in [12,29], which are very close to the second problem. To be more specific, we start by recalling that in [30] was introduced and probed the following assertion: the inequality $a^{a}+b^{b} \geq a^{b}+b^{a}$ holds for all positive real numbers less than or equal to 1 . After that, Cîrtoaje [12] introduced, proved and conjectured several results about inequalities for power-exponential functions. In particular, in [12], it was established that the inequality

$$
a^{r a}+b^{r b} \geq a^{r b}+b^{r a}
$$

\section{Springer}

@2014 Coronel and Huancas; licensee Springer. This is an Open Access article distributed under the terms of the Creative Commons Attribution License (http://creativecommons.org/licenses/by/2.0), which permits unrestricted use, distribution, and reproduction in any medium, provided the original work is properly cited. 
holds true for $r \in[0, e]$ and for either $a \geq b \geq 1 / e$ or $1 / e \geq a \geq b>0$. However, in [12], Cîrtoaje leaves as an open problem the proof of (1.1) for $1>a>1 / e>b>0$. Moreover, in [12] the following conjectures were introduced:

Conjecture 4.3. If $a, b, c$ are positive real numbers, then $a^{2 a}+b^{2 b}+c^{2 c} \geq a^{2 b}+b^{2 c}+c^{2 a}$. Conjecture 4.4. Let $r$ be a positive real number. The inequality

$$
a^{r a}+b^{r b}+c^{r c} \geq a^{r b}+b^{r c}+c^{r a}
$$

holds true for all positive real numbers $a, b, c$ with $a \leq b \leq c$ if and only if $r \leq e$. Conjecture 4.6. Let $r$ be a positive real number. The inequality $a^{r a}+b^{r a} \geq 2$ holds for all nonnegative real numbers $a$ and $b$ if and only if $r \leq 3$.

Conjecture 4.7. If $a$ and $b$ are nonnegative real numbers such that $a+b=2$, then $a^{3 b}+b^{3 a}+2^{-4}(a-b)^{4} \geq 2$.

Conjecture 4.8. If $a$ and $b$ are nonnegative real numbers such that $a+b=1$, then $a^{2 b}+b^{2 a} \leq 1$.

Afterwards, the analysis of (1.1) was completed by Manyama in [14]. Thereafter, of the Cirtoaje conjectures, the milestones of the history are the works of Coronel and Huancas [13], Matejíčka [31], Li [9] and Hisasue [10] (see also the work of Cîrtoaje [29]), where they proved Conjectures 4.3, 4.6, 4.7, and 4.8, respectively. Here, we should be comment that the proof of Conjecture 4.4 is still open. Subsequently, in 2011 Cîrtoaje introduced a new proof of (1.1) and presented the following three new conjectures:

Conjecture 2.1. If $a, b \in] 0,1]$ and $r \in] 0, e]$, then $2 \sqrt{a^{r a} b^{r b}} \geq a^{r b}+b^{r a}$.

Conjecture 2.2. If $a, b, c \in] 0,1]$, then $3 a^{a} b^{b} c^{c} \geq(a b c)^{a}+(a b c)^{b}+(a b c)^{c}$.

Conjecture 5.1. If $a, b$ are nonnegative real numbers satisfying $a+b=1$, and if $k \geq 1$, then $a^{(2 b)^{k}}+b^{(2 a)^{k}} \leq 1$.

Recently, Miyagi and Nishizawa [7] have proved Conjecture 5.1. However, Conjectures 2.1 and 2.2 are still open. Thus, the main focus of this paper are the proofs of Conjectures 4.4, 2.1 , and 2.2.

The main contribution of the present paper is the development of the proof of the following four theorems:

Theorem 1.1 The inequality (1.1) holds, for all positive real numbers $a, b$ and for all $r \in$ $[0, e]$.

Theorem 1.2 The inequality (1.2) holds, for all positive real numbers $a, b, c$ and for all $r \in[0, e]$.

Theorem 1.3 The inequality

$$
2 \sqrt{a^{r a} b^{r b}} \geq a^{r b}+b^{r a}
$$

holds, for all positive real numbers $a, b$ and for all $r \in[0, e]$.

Theorem 1.4 Let $n \in \mathbb{N}$ and $\left.\left.x_{i} \in\right] 0,1\right]^{n}$. Then the inequality

$$
n \prod_{i=1}^{n} x_{i}^{x_{i}} \geq \sum_{i=1}^{n}\left(\prod_{j=1}^{n} x_{j}\right)^{x_{i}}
$$

holds. 
Note that Conjectures 4.4, 2.1, and 2.2 are solved by Theorems 1.2, 1.3, 1.4, respectively. Moreover, we develop a proof of Theorem 1.1 which is an alternative proof of (1.1) for all positive real numbers $a, b$, and $r \in[0, e]$, which is distinct from the existing proofs given in $[14,29]$.

The rest of the paper is organized in two sections: In Section 2 we present the proofs of Theorems 1.1, 1.2, 1.3 and 1.4 and in Section 3 we present some remarks and three new conjectures.

\section{Proofs of main results}

In this section we present the proofs of Theorems 1.1, 1.2, 1.3, 1.4. Firstly, we recall a result of [13]. Then we present the corresponding proofs.

\subsection{A preliminary result}

For completeness and self-contained structure of the proofs of Theorems 1.1 and 1.2, we need the following result of [13].

Proposition 2.1 Consider $s \in \mathbb{R}^{+}$with $s \neq 1, m \in \mathbb{R}^{+}$and $f, g: \mathbb{R}^{+} \rightarrow \mathbb{R}$ defined as follows:

$$
f(t)=t^{s}-t-\gamma^{s}+\gamma \quad \text { and } \quad g(t)= \begin{cases}e^{-\ln (t) /(t-1)}, & t \notin\{0,1\} \\ e^{-1}, & t=1, \\ 0, & t=0 .\end{cases}
$$

Then the following properties are satisfied:

(i) $f(\gamma)=0$ and $f(0)=f(1)=-\gamma^{s}+\gamma$.

(ii) If $s>1, f$ is strictly increasing on $] g(s), \infty$ [ and strictly decreasing on ]0, $g(s)[$.

(iii) If $s \in] 0,1[, f$ is strictly decreasing on $] g(s), \infty$ [ and strictly increasing on $] 0, g(s)[$.

(iv) $g$ is continuous on $\mathbb{R}^{+} \cup\{0\}$ and strictly increasing on $\mathbb{R}^{+}$. Furthermore $y=1$ is a horizontal asymptote of $y=g(t)$.

\subsection{Proof of Theorem 1.1}

Without loss of generality, we assume that $a>b$. Indeed, we find the proof (1.1) by application of Proposition 2.1 with $t=a^{r b}, \gamma=b^{r b}$, and $s=a / b$. Indeed, we distinguish three cases

$\left(\mathrm{a}_{2}\right)$ Case $a>b>1(t>\gamma>1$ and $s>1)$. By Proposition 2.1(iv), we note that $g(s)<1$. Then, by the strictly increasing behavior of $f$ (Proposition 2.1(ii)) we deduce the inequality since:

$$
t>\gamma>1>g(s), \quad s>1 \Rightarrow f(t)=a^{r a}-a^{r b}-b^{r a}+b^{r b}>f(\gamma)=0 .
$$

$\left(\mathrm{b}_{2}\right)$ Case $a>1 \geq b(t>1 \geq \gamma$ and $s>1)$. For $\gamma \in[g(s), 1]$, we conclude the inequality by almost identical arguments to that used before in (i), since $t>1 \geq \gamma \geq g(s)$ and $s>1$. Otherwise, if $\gamma \in[0, g(s)]$, we deduce that

$$
f(t)=a^{r a}-a^{r b}-b^{r a}+b^{r b}>f(1)=f(0)>f(\gamma)=0
$$

which implies the desired inequality. 
$\left(\mathrm{c}_{2}\right)$ Case $1>a>b>0(1>t>\gamma>0$ and $s>1)$. First, we define $h:[0,1] \rightarrow \mathbb{R}$ by the correspondence rule $h(t)=-r t \ln t$ for $t>0$ and $h(0)=0$. The function $h$ is concave and has a maximum at $(1 / e, r / e)$. Thus, we deduce that

$-r b \ln b<1, \quad$ for all $b \in[0,1]$ and $r \in[0, e]$.

Secondary, by the Napier inequality [32]

$$
0<b<a \Rightarrow \frac{1}{a}<\frac{\ln a-\ln b}{a-b}<\frac{1}{b} .
$$

From (2.1) and (2.2) we have

$$
-r b \ln b \leq 1 \leq \frac{1}{a}<\frac{\ln a-\ln b}{a-b}
$$

which implies $\gamma>g(s)$. The proof of this case is completed by application of Proposition 2.1(ii).

Hence, by $\left(a_{2}\right),\left(b_{2}\right)$, and $\left(c_{2}\right)$ we conclude that Theorem 1.1 is valid.

\subsection{Proof of Theorem 1.2}

The proof of this theorem is again developed by application of Proposition 2.1. Firstly, we recall the notation of [13]:

$$
\begin{aligned}
& \mathbb{R}_{+}^{3}=\left\{(a, b, c) \in \mathbb{R}^{3} / a>0, b>0 \text { and } c>0\right\}, \\
& \mathbb{E}_{1}=\left\{(a, b, c) \in \mathbb{R}_{+}^{3} / a=b=c \text { or } a=b \neq c \text { or } a \neq b=c\right\} \\
& \mathbb{E}_{a}^{+}=\left\{(a, b, c) \in \mathbb{R}_{+}^{3} / a \geq 1 \text { and } a>\max \{b, c\}\right\}, \\
& \mathbb{E}_{a}^{-}=\left\{(a, b, c) \in \mathbb{R}_{+}^{3} / 1>a>\max \{b, c\}\right\} \\
& \mathbb{E}_{b}^{+}=\left\{(a, b, c) \in \mathbb{R}_{+}^{3} / b \geq 1 \text { and } b>\max \{a, c\}\right\}, \\
& \mathbb{E}_{b}^{-}=\left\{(a, b, c) \in \mathbb{R}_{+}^{3} / 1>b>\max \{a, c\}\right\}, \\
& \mathbb{E}_{c}^{+}=\left\{(a, b, c) \in \mathbb{R}_{+}^{3} / c \geq 1 \text { and } c>\max \{a, b\}\right\} \text { and } \\
& \mathbb{E}_{c}^{-}=\left\{(a, b, c) \in \mathbb{R}_{+}^{3} / 1>c>\max \{a, b\}\right\} .
\end{aligned}
$$

The family $\left\{\mathbb{E}_{1}, \mathbb{E}_{a}^{+}, \mathbb{E}_{a}^{-}, \mathbb{E}_{b}^{+}, \mathbb{E}_{b}^{-}, \mathbb{E}_{c}^{+}, \mathbb{E}_{c}^{-}\right\}$is a set partition of $\mathbb{R}_{+}^{3}$. Now, with this notation, we subdivide the proof in three parts:

$\left(\mathrm{a}_{3}\right)$ Case $(a, b, c) \in \mathbb{E}_{1}$. This special case is a direct consequence of Theorem 1.1.

$\left(\mathrm{b}_{3}\right)$ Case $(a, b, c) \in \mathbb{E}_{a}^{+} \cup \mathbb{E}_{b}^{+} \cup \mathbb{E}_{c}^{+}$. If $(a, b, c) \in \mathbb{E}_{a}^{+}$, we apply Theorem 1.1 and Proposition 2.1 as follows. We select $t=a^{r b}, \gamma=c^{r b}$ and $s=a / b$, the monotonic behavior and properties of function $f$, defined on Proposition 2.1, imply that

$$
a^{r a}+c^{r b}>a^{r b}+c^{r a}
$$

since $t>\gamma, t>1$ and $s>1$. Indeed, the corresponding proof of (2.3) needs the distinction of two cases: $c \geq 1$ and $c<1$. If $c \geq 1$, then $\gamma>1$ and $\gamma \in] g(s), \infty[$, so $f$ is strictly increasing and $t>\gamma$ implies (2.3). For $c<1$, we note that $\gamma<1$ and $-\gamma^{s}+\gamma \geq 0$ since $s>1$ and $\left.1 \in\right] g(s), \infty[$, 
then the assumption $t>1$ implies that $f(t)>f(1)=-\gamma^{s}+\gamma \geq 0=f(\gamma)$ and (2.3) is again true for this subcase. Moreover, for $(a, b, c) \in \mathbb{E}_{a}^{+} \subset \mathbb{R}_{+}^{3}$, by Theorem 1.1, we recall that the inequality

$$
c^{r c}+b^{r b}>b^{r c}+c^{r b}
$$

holds true for all $r \in[0, e]$. Adding (2.3) and (2.4) we deduce (1.2).

The proof for $(a, b, c) \in \mathbb{E}_{b}^{+} \cup \mathbb{E}_{c}^{+}$is similar to the case $(a, b, c) \in \mathbb{E}_{a}^{+}$and we omit the details. However, we comment that for $(a, b, c) \in \mathbb{E}_{b}^{+}$we choose $t=b^{r c}, \gamma=c^{2 c}$, and $s=b / c$; and for $(a, b, c) \in \mathbb{E}_{c}^{+}$we select $t=c^{r a}, \gamma=b^{r a}$, and $s=c / a$.

$\left(c_{3}\right)$ Case $(a, b, c) \in \mathbb{E}_{a}^{-} \cup \mathbb{E}_{b}^{-} \cup \mathbb{E}_{c}^{-}$. Without loss of generality, we assume that $(a, b, c) \in \mathbb{E}_{a}^{-}$ is such that $c<b<a$, since the proof for $b<c<a$ is similar. We note that $\Omega=[0, e] \times[0,1]$ can be partitioned in the two sets

$$
\begin{aligned}
& \Omega_{1}=\left\{(r, c) \in \Omega: c \in\left[(r-1) r^{-1}, 1\right]\right\} \text { and } \\
& \Omega_{2}=\left\{(r, c) \in \Omega: c \in\left[0,(r-1) r^{-1}\right]\right\} .
\end{aligned}
$$

Now, we continue the proof by distinguish the following two subcases: $(r, c) \in \Omega_{1}$ and $(r, c) \in \Omega_{2}$.

For the subcase $(r, c) \in \Omega_{1}$, we apply the function $f$ given on Proposition 2.1 with $t=b^{r c}$, $\gamma=c^{r c}$, and $s=a / c$ to prove

$$
b^{r a}+c^{r c}>b^{r c}+c^{r a} \quad \text { for } 0<c<b<a<1 \text { and }(r, c) \in \Omega_{1} .
$$

Indeed, we firstly note that the function $m:[c, 1] \rightarrow \mathbb{R}$ defined as follows: $m(z)=z c^{r z}-c^{r c+1}$ has the following properties:

$\left(\mathrm{m}_{a}\right) m(c)=0$

$\left(\mathrm{m}_{b}\right) \quad m(1)=c^{r}\left(1-c^{r c+1-r}\right) \geq 0$ for all $(r, c) \in \Omega_{1}$ since $c>(r-1) / r$; and

$\left(\mathrm{m}_{c}\right) \quad m$ has a maximum at $z_{\max }=-1 / r \ln c$, since the first and second derivatives of $m$ are given by $m^{\prime}(z)=c^{r z}(1+r z \ln c)$ and $m^{\prime \prime}(z)=c^{r z}(2 r+r z \ln c) \ln c$ and naturally $m^{\prime}\left(z_{\max }\right)=$ 0 and $m^{\prime \prime}\left(z_{\max }\right)<0$.

Moreover, we notice that $z_{\max } \geq c$ is equivalent to $1>-r c \ln c$, which is true for $r \in[0, e]$ and $c \in[0,1]$; see the proof of $(2.1)$. Then, by $\left(\mathrm{m}_{a}\right)-\left(\mathrm{m}_{c}\right)$, it follows that $m(z) \geq 0$, for all $z \in[c, 1]$. In particular, for $z=a$, we have

$$
a c^{r a}>c^{r c+1}, \quad \text { for } a \in[c, 1] \subset[0,1] \text { and } r \in[0, e] .
$$

Now, from (2.6), we note that

$$
\begin{aligned}
& a c^{r a}>c^{r c+1} \Rightarrow c^{r(a-c)}>\frac{c}{a} \Rightarrow r c \ln c>\frac{c \ln (c / a)}{a-c} \\
& \Rightarrow \quad c^{r c}>e^{\frac{-c \ln (a / c)}{a-c}} \Rightarrow \gamma>g(s),
\end{aligned}
$$

which implies (2.5) by application of Proposition 2.1(ii), since $t>\gamma>g(s)$ and $f$ is increasing on $] g(s), \infty[$. 
For the subcase $(r, c) \in \Omega_{2}$, we apply the function $f$ given on Proposition 2.1 with $t=b^{r c}$, $\gamma=c^{r c}$, and $s=a / c$ to prove

$$
b^{r a}+c^{r c}>b^{r c}+c^{r a} \text { for } 0<c<b<a<1 \text { and }(r, c) \in \Omega_{2} .
$$

We note that the inequality $c^{r c}>c^{r-1}$ holds true for all $(r, c) \in \Omega_{2}$. Now, in order to deduce that $\gamma>g(s)$ it is sufficient to prove that $c^{r-1}>g(s)$. Indeed, the function $q:[c, 1] \rightarrow \mathbb{R}$ defined as $q(z)=c^{(1-r) z} z^{c}-c^{c+c(1-r)}$ has the following properties:

$\left(\mathrm{q}_{a}\right) q(c)=0$

( $\left.\mathrm{q}_{b}\right) q(1)=c^{1-r}\left(1-c^{c+(c-1)(1-r)}\right) \geq 0$ for all $(r, c) \in \Omega_{2}$, since $c \in[0,(r-1) / r]$; and

$\left(\mathrm{q}_{c}\right) q$ is increasing in $[c, 1]$.

Then we deduce that $q(z) \geq 0$ for all $z \in[c, 1]$. In particular, for $z=a \in[c, 1]$, we deduce that $c^{(1-r) a} a^{c}-c^{c+c(1-r)} \geq 0$, which implies the following sequence of implications:

$$
c^{(1-r) a} a^{c}>c^{c+c(1-r)} \Rightarrow \frac{c^{(1-r) a}}{c^{c(1-r)}}>\frac{c^{c}}{a^{c}} \Rightarrow c^{1-r}>g(s) .
$$

Thus (2.8) holds true.

From (2.5) and (2.8), we deduce that

$$
b^{r a}+c^{r c}>b^{r c}+c^{r a} \text { for } 0<c<b<a<1 \text { and } r \in[0, e] .
$$

Hence, to complete the proof for $0<c<b<a<1$, we add the inequality (2.9) with $a^{r a}+$ $b^{r b}>a^{r b}+b^{r a}$ for $r \in[0, e]$, which is true by Theorem 1.1.

For $(a, b, c) \in \mathbb{E}_{b}^{-} \cup \mathbb{E}_{c}^{-}$we can follow line by line the proof of $(a, b, c) \in \mathbb{E}_{a}^{-}$. However, we can obtain a direct proof by applying the result obtained for $(a, b, c) \in \mathbb{E}_{a}^{-}$by interchanging the role of variables. For instance, if $(a, b, c) \in \mathbb{E}_{b}^{-}$then $(b, a, c) \in \mathbb{E}_{a}^{-}$, which implies (1.2).

Hence, by $\left(a_{3}\right),\left(b_{3}\right)$, and $\left(c_{3}\right)$ we have the complete proof of Theorem 1.2.

\subsection{Proof of Theorem 1.3}

Given $b \in] 0,1]$, we define the function $H:] 0,1] \rightarrow \mathbb{R}$ as follows:

$$
H(x)=2 \sqrt{x^{r x} b^{r b}}-b^{r x}-x^{r b} .
$$

Then we prove that $H(x)>0$ for all $x \in] 0,1]$, which naturally implies the inequality $2 \sqrt{a^{r a} b^{r b}} \geq a^{r b}+b^{r a}$ for $x=a$. Indeed, we prove that the function $H$ has a global minimum at $x=b$. The fact that in $x=b$ there is a local minimum of $H$ follows by noticing that $H^{\prime}(b)=0$ and $H^{\prime \prime}(b)>0$, since

$$
\begin{aligned}
& H^{\prime}(x)=r\left[\sqrt{x^{r x} b^{r b}}(\ln x+1)-b^{r x} \ln b-b x^{r b-1}\right] \text { and } \\
& H^{\prime \prime}(x)=r\left[\sqrt{x^{r x} b^{r b}}\left\{r(\ln x+1)^{2}+x^{-1}\right\}-r b^{r x}(\ln b)^{2}-b(r b-1) x^{r b-2}\right] .
\end{aligned}
$$

Meanwhile, the property that $b$ is a global minimum of $H$ can be proved by rewriting $H^{\prime}$ as the difference of two functions and by analyzing the sign of $H^{\prime}$ using some properties 
of this new functions. Indeed, to be more specific, we note that $H^{\prime}(x)=r[K(x)-Q(x)]$ for all $x \in] 0,1]$, where the functions $K$ and $Q$ are defined as follows:

$$
K(x)=\sqrt{x^{r x} b^{r b}}(\ln x+1) \text { and } Q(x)=b^{r x} \ln b+b x^{r b-1} .
$$

The functions $K$ and $Q$ have the following properties:

$\left(K_{1}\right) K$ is strictly increasing on $\left.] 0,1\right]$, since $K^{\prime}(x)=\sqrt{x^{r x} b^{r b}}\left\{r(\ln x+1)^{2}+x^{-1}\right\}>0$, for all $x \in] 0,1]$.

$\left(K_{2}\right) K(x) \rightarrow-\infty$ when $x \rightarrow 0^{+}, K(1 / e)=0$, and $K(1)=\sqrt{b^{r b}}$.

$\left(\mathrm{Q}_{1}\right)$ The derivative of $Q$ is given by $Q^{\prime}(x)=r b^{r x}(\ln b)^{2}+b(r b-1) x^{r b-2}$, for all $\left.\left.x \in\right] 0,1\right]$. Then, in order to analyze the sign of $Q^{\prime}$, we introduce the set $\left.\left.\left.\left.\Lambda=\right] 0,1\right] \times\right] 0, e\right]$ and a partition $\left\{\Lambda_{1}, \Lambda_{2}, \Lambda_{3}\right\}$ of $\Lambda$, where

$$
\begin{aligned}
& \left.\left.\Lambda_{1}=\left\{(b, r) \in \Lambda: Q^{\prime}(x)>0 \text { for all } x \in\right] 0,1\right]\right\}, \\
& \left.\left.\Lambda_{2}=\left\{(b, r) \in \Lambda: Q^{\prime}(x)<0 \text { for all } x \in\right] 0,1\right]\right\}, \\
& \left.\left.\Lambda_{3}=\{(b, r) \in \Lambda: \exists ! c \in] 0,1\right] \text { such that } Q \text { has a minimum at } x=c\right\} .
\end{aligned}
$$

We note that the sets $\Lambda_{i}, i=1,2,3$, are not empty since for instance $] 0,1\left[\times[1 / b, e] \subset \Lambda_{1}\right.$ for all $b \in] 0,1],\{1\} \times] 0,1\left[\subset \Lambda_{2}\right.$ and $] 0,1\left[\times\{1\} \subset \Lambda_{3}\right.$. Moreover, we note that $r b>1$ implies that $(b, r) \in \Lambda_{1}$ and naturally $\Lambda_{2} \cup \Lambda_{3}$ is a subset of $\left.\left.] 0,1\right] \times\right] 0,1 / b[$. The uniqueness of $c$ can be deduced by noticing that the solution of $Q^{\prime}(x)=0$ is equivalent to the intersection of the following two monotone functions: $S(x)=r b^{r x}(\ln b)^{2}$ and $J(x)=b(1-r b) x^{r b-2}$.

$\left(\mathrm{Q}_{2}\right) Q(x) \rightarrow \ln b$ when $x \rightarrow 0^{+}$, and $Q(1)=b^{r} \ln b+b$.

From $\left(\mathrm{K}_{1}\right)$ and $\left(\mathrm{Q}_{1}\right)$ we deduce the uniqueness of $\left.\left.b \in\right] 0,1\right]$ such that $Q(b)=K(b)$ or equivalently $H^{\prime}(b)=0$. Now, from $\left(K_{2}\right)$ and $\left(\mathrm{Q}_{2}\right)$, we note that $Q\left(0^{+}\right)>K\left(0^{+}\right)$for all $(r, b) \in \Lambda$ since $K\left(0^{+}\right)=-\infty$. Then $H^{\prime}(x)<0$ for all $\left.x \in\right] 0, b$. Additionally, from $\left(\mathrm{K}_{2}\right)$ and $\left(\mathrm{Q}_{2}\right)$, we observe that $Q(1)<K(1)$. This fact is a consequence of the fact that the function $F(w, r)=$ $\sqrt{w^{r w}}-w^{r} \ln (w)-w$ is strictly decreasing in $r$, since $F_{r}(w, r)=\ln (w)\left((r / 2) \sqrt{w^{r w}}-w^{r} \ln (w)\right)<$ 0 . Consequently, for $r<e$ we have $F(w, r)>F(w, e)=\sqrt{w^{e w}}-w^{e} \ln (w)-w>0$ for all $w \in] 0,1]$. Hence, for $w=b$ we get $F(b, r)>0$ or $Q(1)<K(1)$, which implies that $H^{\prime}(x)>0$ for all $x \in] b, 1]$. Thus, $b$ is a global minimum of $H$. Therefore, $H(x) \geq H(b)=0$ for all $x \in] 0,1]$ and in particular for $x=a$.

\subsection{Proof of Theorem 1.4}

The proof follows by the fact that the function $P:] 0,1]^{n-1} \rightarrow \mathbb{R}$ is defined by the following correspondence rule:

$$
\left.\left.P\left(z_{1}, \ldots, z_{n-1}\right)=n x_{n}^{x_{n}} \prod_{i=1}^{n} z_{i}^{z_{i}}-\left(x_{n} \prod_{j=1}^{n-1} z_{j}\right)^{x_{n}}-\sum_{i=1}^{n-1}\left(x_{n} \prod_{j=1}^{n-1} z_{j}\right)^{x_{i}}, \quad x_{n} \in\right] 0,1\right]
$$

and it has a global minimum at $\left(z_{1}, \ldots, z_{n-1}\right)=\left(x_{n}, \ldots, x_{n}\right)$. Indeed, for simplicity of notation we develop the details of the proof for $n=3$ and with $\left(x_{1}, x_{2}, x_{3}\right)=(a, b, c)$. Note that, in this case for an arbitrary $c \in] 0,1]$, the function $P:] 0,1]^{2} \rightarrow \mathbb{R}$ has the following form:

$$
P(x, y)=3 x^{x} y^{y} c^{c}-(x y c)^{x}-(x y c)^{y}-(x y c)^{c} .
$$


Then we have

$$
\begin{aligned}
P_{x}(x, y)= & 3 x^{x} y^{y} c^{c}(\ln (x)+1)-(\ln (x y c)+1)(x y c)^{x}-\frac{y}{x}(x y c)^{y}-\frac{c}{x}(x y c)^{c}, \\
P_{y}(x, y)= & 3 x^{x} y^{y} c^{c}(\ln (y)+1)-\frac{x}{y}(x y c)^{x}-(\ln (x y c)+1)(x y c)^{y}-\frac{c}{y}(x y c)^{c}, \\
P_{x x}(x, y)= & 3 x^{x} y^{y} c^{c}\left[\frac{1}{x}+(\ln (x)+1)^{2}\right] \\
& -\left[\frac{1}{x}+(\ln (x y c)+1)^{2}\right](x y c)^{x}-\left[\frac{y^{2}-y}{x^{2}}\right](x y c)^{y}-\left[\frac{c^{2}-c}{x^{2}}\right](x y c)^{c}, \\
P_{y y}(x, y)= & 3 x^{x} y^{y} c^{c}\left[\frac{1}{y}+(\ln (y)+1)^{2}\right] \\
& -\left[\frac{x^{2}-x}{y^{2}}\right](x y c)^{x}-\left[\frac{1}{y}+(\ln (x y c)+1)^{2}\right](x y c)^{y}-\left[\frac{c^{2}-c}{y^{2}}\right](x y c)^{c}, \\
& -\left[\frac{x(\ln (x y c)+1)+1}{y}\right](x y c)^{x}-\left[\frac{y(\ln (x y c)+1)+1}{x}\right](x y c)^{y}-\left[\frac{c^{2}}{x y}\right](x y c)^{c} .
\end{aligned}
$$

An evaluation at $(c, c)$ implies that

$$
\begin{aligned}
& P_{x}(c, c)=P_{y}(c, c)=0, \\
& P_{x x}(c, c)=P_{y y}(c, c)=c^{3 c-1}\left(-6 c(\ln (c))^{2}+4\right), \\
& P_{x y}(c, c)=P_{y x}(c, c)=c^{3 c-1}\left(3 c(\ln (c))^{2}-2\right) .
\end{aligned}
$$

Now, defining $\mathbb{P}_{1}(w)=-6 w(\ln (w))^{2}+4$ and $\mathbb{P}_{2}(w)=27 w^{2}(\ln w)^{4}-24 w(\ln w)^{2}+4$, we observe that $P_{x x}(c, c)=c^{3 c-1} \mathbb{P}_{1}(c)$ and $P_{x x}(c, c) P_{y y}(c, c)-P_{x y}(c, c) P_{x y}(c, c)=c^{2(3 c-1)} \mathbb{P}_{2}(c)$. Then the Hessian matrix associated to $P$ at $(c, c)$ is positive semidefinite since both functions, $\mathbb{P}_{1}$ and $\mathbb{P}_{2}$, are positive on $\left.] 0,1\right]$ or equivalently the function $P$ has a local minimum at $(c, c)$. Now, we deduce that $(c, c)$ is the global minimum since we can prove that $(c, c)$ is the unique solution of $\left(P_{x}, P_{y}\right)=(0,0)$. Indeed, assuming that there is $(x, y)$ with $x \neq y \neq c$ such that $P_{x}(x, y)=P_{y}(x, y)=0$, we can deduce a contradiction. Note that

$$
\begin{aligned}
0 & =\left|P_{x}(x, y)-P_{y}(x, y)\right| \\
& \geq\left|\min \left\{3 x^{x} y^{y} c^{c},(x y c)^{x},(x y c)^{y},(x y c)^{c}\right\}\right|\left|\ln \left(\frac{x}{y}\right)-\frac{y}{x}-\frac{c}{x}+\frac{x}{y}+\frac{c}{y}\right| \\
& \geq\left|\min \left\{3 x^{x} y^{y} c^{c},(x y c)^{x},(x y c)^{y},(x y c)^{c}\right\}\right|\left|\frac{1}{x}+\frac{x+y}{x y}+\frac{c}{x y}\right||x-y|,
\end{aligned}
$$

since the inequality $\ln (r)>(r-1) / r$ holds for all $r>0$ and $r \neq 1$ (see for instance [27]). Then $x=y$, which is a contradiction with the assumption that $x \neq y$. Thus, we see that $(c, c)$ is a global minimum of the function $P$ or equivalently $P(x, y) \geq P(c, c)=0$ for all $(x, y) \in] 0,1]^{2}$, which implies the desired inequality for $(x, y)=(a, b)$. 


\section{Additional remarks on possible generalizations}

In this section we present the possible extensions of Theorems 1.1, 1.2, and 1.3 to a sequence of positive real numbers. We note that the natural generalizations of (1.2) and (1.3) are given by

$$
\begin{aligned}
& \sum_{i=1}^{n} x_{i}^{r x_{i}} \geq x_{n}^{r x_{1}}+\sum_{i=1}^{n-1} x_{i}^{r x_{i+1}}, \quad\left(x_{1}, \ldots, x_{n}\right) \in \mathbb{R}_{+}^{n}, r \in[0, e], \\
& \left.\left.\sqrt[n]{\prod_{i=1}^{n} x_{i}^{r x_{i}}} \geq x_{n}^{r x_{1}}+\sum_{i=1}^{n-1} x_{i}^{r x_{i+1}}, \quad\left(x_{1}, \ldots, x_{n}\right) \in\right] 0,1\right]^{n},
\end{aligned}
$$

respectively. We present a partial proof of (3.1) (see Lemma 3.1, below) and leave as a conjecture the proof of (3.2).

Lemma 3.1 The inequality given in (3.1) holds for all $r \in[0, e]$, if we restrict $\left(x_{1}, \ldots, x_{n}\right)$ to the hypercube $[0,1]^{n}$.

Proof Before we start the proof, we notice that the function $\Upsilon(x, y)=x^{a / b}-x-y^{a / b}+y$ defined from $\mathbb{R}_{+}^{2} \rightarrow \mathbb{R}$ and for $a>b$ is concave and $\Upsilon(0,0)=\Upsilon(1,0)=\Upsilon(0,1)=\Upsilon(1,1)=0$. Then $\Upsilon(x, y) \geq 0$ for all $(x, y) \in[0,1] \times[0,1]$. Similarly, the function $\Upsilon_{s}(w, z)=\Upsilon(z, w)$ for $a<b$ is concave and $\Upsilon_{s}(w, z) \geq 0$ for all $(w, z) \in[0,1] \times[0,1]$. Now, we proceed by induction on $n$. Let us assume that the theorem is valid for a sequence of positive numbers $\left(x_{1}, \ldots, x_{k}\right)$ for all $k<n$. We note that

$$
\begin{aligned}
\sum_{i=1}^{n} x_{i}^{r x_{i}}-x_{n}^{r x_{1}}-\sum_{i=1}^{n-1} x_{i}^{r x_{i+1}}= & {\left[\sum_{i=1}^{n-1} x_{i}^{r x_{i}}-x_{n-1}^{r x_{1}}-\sum_{i=1}^{n-2} x_{i}^{r x_{i+1}}\right]+\left[x_{n}^{r x_{n}}+x_{n-1}^{r x_{n-1}}-x_{n}^{r x_{n-1}}-x_{n-1}^{r x_{n}}\right] } \\
& +\left[x_{n}^{r x_{n-1}}-x_{n}^{r x_{1}}-x_{n-1}^{r x_{n-1}}+x_{n-1}^{r x_{1}}\right] \\
:= & \mathbb{K}_{1}+\mathbb{K}_{2}+\mathbb{K}_{3} .
\end{aligned}
$$

The terms $\mathbb{K}_{1}$ and $\mathbb{K}_{2}$ are positive by the inductive hypothesis. Meanwhile, the term $\mathbb{K}_{3}$ is positive by the concavity of the functions $\Upsilon$ and $\Upsilon_{s}$. Note that $a=x_{n-1}$ and $b=x_{n}$, and $\mathbb{K}_{3}=\Upsilon\left(x_{n}^{r x_{1}}, x_{n-1}^{r x_{1}}\right)$ or $\mathbb{K}_{3}=\Upsilon_{s}\left(x_{n-1}^{r x_{1}}, x_{n}^{r x_{1}}\right)$, depending if $x_{n-1}>x_{1}$ or $x_{n-1}<x_{1}$, respectively. Then, by (3.3), it follows that the lemma is valid.

Conjecture 3.1 Let $n \in \mathbb{N}$ and $n>4$. Then the inequality (3.1) holds for all $\left(x_{1}, \ldots, x_{n}\right) \in \mathbb{R}_{+}^{n}$ and $r \in[0, e]$.

Conjecture 3.2 Let $n \in \mathbb{N}$ and $n \geq 3$. Then the inequality (3.2) holds for all $r \in[0, e]$.

Conjecture 3.3 Let $n \in \mathbb{N}$ and $\left.\left.x_{i} \in\right] 0,1\right]^{n}$. Then the inequality

$$
n \prod_{i=1}^{n} x_{i}^{r x_{i}} \geq \sum_{i=1}^{n}\left(\prod_{j=1}^{n} x_{j}\right)^{r x_{i}}
$$

holds for all $r \in[0, e]$. 


\section{Competing interests}

The authors declare that they have no competing interests.

\section{Authors' contributions}

All authors contributed equally to the writing of this paper. All authors read and approved the final manuscript.

\section{Acknowledgements}

We acknowledge the support of 'Univesidad del Bío-Bío' (Chile) through the research projects 124109 3/R, $10470901 \mathrm{~F} / \mathrm{E}$, and $121909 \mathrm{Gl} / \mathrm{C}$

\section{Received: 6 September 2014 Accepted: 3 December 2014 Published: 12 Dec 2014}

\section{References}

1. Dahmani, A, Karima Belaide, K: Exponential inequalities in calibration problems with gaussians errors. Commun. Stat. Theory Methods 42(19), 3596-3607 (2013)

2. Hou, Q, Lin, Z, Dusing, RW, Gajewski, BJ, Mccallum, RW: A bayesian hierarchical assessment of gastric emptying with the linear, power exponential and modified power exponential models. Neurogastroenterology \& Motility 22(12), 1308-1317 (2010)

3. Floyd, BNI, Camilleri, M, Andresen, V, Esfandyari, T, Busciglio, I, Zinsmeister, AR: Comparison of mathematical methods for calculating colonic compliance in humans: power exponential, computer-based and manual linear interpolation models. Neurogastroenterology \& Motility 20(4), 330-335 (2008)

4. Park, J-S, Baek, J: Efficient computation of maximum likelihood estimators in a spatial linear model with power exponential covariogram. Comput. Geosci. 27(1), 1-7 (2001)

5. Bruno, AD: Power-exponential expansions of solutions to an ordinary differential equation. Dokl. Math. 85(3), 336-340 (2012)

6. Fan, X, Grama, I, Liu, Q: Large deviation exponential inequalities for supermartingales. Electron. Commun. Probab. 17(59), 1-8 (2012)

7. Miyagi, M, Nishizawa, Y: A short proof of an open inequality with power-exponential functions. Aust. J. Math. Anal. Appl. 11(1), 6 (2014)

8. Miyagi, M, Nishizawa, Y: Proof of an open inequality with double power-exponential functions. J. Inequal. Appl. 2013, 468 (2013)

9. Li, Y: Solutions of two conjectures on inequalities with power-exponential functions. RGMIA Res. Rep. Collect. 12(4), 7 (2009)

10. Hisasue, M: Solution of inequalities with power-exponential functions by Cîrtoaje. Aust. J. Math. Anal. Appl. 9(2), 4 (2012)

11. Cîrtoaje, V: Proofs of three open inequalities with power-exponential functions. J. Nonlinear Sci. Appl. 4(2), 130-137 (2011)

12. Cîrtoaje, V: On some inequalities with power-exponential functions. J. Inequal. Pure Appl. Math. 10(1), 21 (2009)

13. Coronel, A, Huancas, F: On the inequality $a^{2 a}+b^{2 b}+c^{2 c} \geq a^{2 b}+b^{2 c}+c^{2 a}$. Aust. J. Math. Anal. Appl. 9(1), $3(2012)$

14. Manyama, S: Solution of one conjecture on inequalities with power-exponential functions. Aust. J. Math. Anal. Appl. 7(2), $1(2010)$

15. Matejíčka, L: On an open problem posed in the paper 'Inequalities of power-exponential functions'. J. Inequal. Pure Appl. Math. 9(3), 75 (2008)

16. Qi, F, Debnath, L: Inequalities for power-exponential functions. J. Inequal. Pure Appl. Math. 1(2), 15 (2000)

17. Qi, F, Xu, S-L: The function $\left(b^{x}-a^{x}\right) / x$ : inequalities and properties. Proc. Am. Math. Soc. 126(11), 3355-3359 (1998)

18. Cerone, P, Dragomir, SS: Mathematical Inequalities. CRC Press, Boca Raton (2011)

19. Wright, EM: Solution of the equation $z e^{z}=a$. Proc. R. Soc. Edinb., Sect. A 65, 193-203 (1959)

20. Wright, EM: Solution of the equation $z e^{z}=a$. Bull. Am. Math. Soc. 65, 89-93 (1959)

21. Wright, EM: Solution of the equation $(p z+q) e^{z}=r z+s$. Bull. Am. Math. Soc. 66, 277-281 (1960)

22. Lambert, JH: Observations variae in mathesin puram. Acta Helvitica, Physico-Mathematico-Anatomico-Botanico-Medica 63, 128-168 (1758)

23. Euler, J: De serie lambertina plurimisque eius insignibus proprietatibus. Acta Acad. Scient. Petropol. 2, 29-51 (1783)

24. Pólya, G, Szegő, G: Aufgaben und Lehrsätze aus der Analysis. Band II: Funktionentheorie, Nullstellen, Polynome Determinanten, Zahlentheorie. Springer, Berlin (1971)

25. Corless, RM, Gonnet, GH, Hare, DEG, Jeffrey, DJ, Knuth, DE: On the Lambert W function. Adv. Comput. Math. 5(4), 329-359 (1996)

26. Hoorfar, A, Hassani, M: Inequalities on the Lambert $W$ function and hyperpower function. J. Inequal. Pure Appl. Math. 9(2), 51 (2008)

27. Bullen, PS: A Dictionary of Inequalities. Pitman Monographs and Surveys in Pure and Applied Mathematics, vol. 97. Longman, Harlow (1998)

28. Luo, J, Wen, JJ: A power-mean discriminance of comparing $a^{b}$ and $b^{a}$. In: Yand, X-Z (ed.) Research Inequalities, pp. 83-88. People's Press of Tibet, The People's Republic of China (2000)

29. Cîrtoaje, V: Proofs of three open inequalities with power-exponential functions. J. Nonlinear Sci. Appl. 4(2), 130-137 (2011)

30. zeikii, A, Cîrtoaje, V, Berndt, B:. Mathlinks Forum. http://www.mathlinks.ro/Forum/viewtopic.php?t=118722 (2006) Accessed November 2006

31. Matejíčka, L: Solution of one conjecture on inequalities with power-exponential functions. J. Inequal. Pure Appl. Math. 10(3), 1-5 (2009)

32. Nelsen, RB: Napier's inequality (two proofs). Coll. Math. J. 24(2), 165 (1993)

10.1186/1029-242X-2014-509

Cite this article as: Coronel and Huancas: The proof of three power-exponential inequalities. Journal of Inequalities and Applications 2014, 2014:509 